\title{
ARTIGOS
}

\section{SOUFFRANCE ETHIQUE ET SOUFFRANCE TRAGIQUE : L'ELABORATION LEVINASSIENNE DE LA CRITIQUE NIETZSCHEENNE DE LA COMPASSION}

\author{
Aïcha Liviana Messina* \\ alivianamessina@gmail.com
}

RÉSUMÉ Cet article analyse le problème de la souffrance dans l'œuvre de Lévinas à la lumière de la critique nietzschéenne de la compassion. Il $s$ 'agit dans un premier temps de montrer que, bien que la description que fait Lévinas de la souffrance éthique (le souffrir pour Autrui) soit similaire à l'idée nietzschéenne de la souffrance tragique ou inutile, les premiers écrits de Lévinas se concentrent aussi sur les dangers politiques qui resultent de la conception nietzschéenne du corps et de sa vision tragique de la souffrance. Dans un second temps, cet article se propose de montrer que la souffrance éthique telle que la décrit Lévinas ne correspond pas à la façon dont Nietzsche décrit la compassion comme étant une forme de nihilisme (au sens d'une négation de la vie). Il soutient au contraire l'idée que la souffrance éthique chez Lévinas permet d'aller plus loin dans la conception qu'a Nietzsche de l'amitié et de la vie.

Mots clés Lévinas, Nietzsche, compassion, corps, vie.

ABSTRACT This article analyses the problem of suffering in Lévinas's work in the light of Nietzsche's critic of compassion. It argues, in a first step, that although Lévinas's description of ethical suffering (namely, the idea of suffering for the other) is similar to Nietzsche's description of suffering as 
tragic or useless, Lévinas's first writings also focus on the political dangers associated with Nietzsche's conception of the body and of a purely tragic conception of suffering. In a second step, this article shows that the question of ethical suffering, such as Lévinas describes it, does not match Nietzsche's conception of compassion as being a form of nihilism (conceived as a negation of life), but, on the contrary, allows for the radicalization of some of Nietzsche's views on friendship and of life.

Keywords: Lévinas, Nietzsche, Suffering, Compassion, Body, Life.

Le XXème siècle aura sans doute été le siècle où la souffrance a cessé d'être un simple objet de la pensée pour devenir le problème de la pensée elle-même. Le désastre qu'a représenté la seconde guerre a en effet confronté la pensée à l'injustifiable, à ce dont la pensée ne pouvait plus s'emparer en lui donnant sens. En ce sens, comme l'écrit Lévinas dans « La souffrance inutile», la souffrance a cela de révolutionnaire qu'elle marque la fin de la théodicée. ${ }^{1}$ Elle interdit par elle-même, par le type de phénomène qu'elle constitue, toute tentative d'expliquer le mal et par là de le justifier. Mais si la « souffrance inutile » aura été le mal du siècle, l'inutilité de celle-là ne se réduit pas à des cas particuliers ou extraordinaires de souffrance. Pour Lévinas, la souffrance est cela même qui se produit comme refus du sens. ${ }^{2}$ Dans sa passivité, la souffrance échappe à la dynamique du sens, à l'activité de l'esprit dont l'agir sa possibilité - est précisément le sens. Ainsi, loin d'être un objet de la pensée, la souffrance pourrait être ce qui marque l'échec de cette dernière. Se refusant au sens, la souffrance n'est pas seulement impensable : elle met la pensée (sa possibilité) en question. Elle pourrait signifier le désastre de la pensée. A moins, comme le suggère Blanchot dans «L'écriture du désastre », qu'elle ne

1 LEVINAS, E. «La souffrance inutile » (dorénavant abrégé SI). In : « Entre nous. Essais sur le penser-àl'autre ». Paris : Grasset, 1991 : «C'est peut-être le fait le plus révolutionnaire de notre conscience du XXème siècle - mais aussi un événement de l'Histoire Sainte - que la destruction de tout équilibre entre la théodicée explicite et implicite de la pensée occidentale et les formes que la souffrance et son mal puisent dans le déroulement même de ce siècle », p. 114.

2 Pour Lévinas, la souffrance est « reniement et refus de sens s'imposant comme qualité sensible » (ibid., p. 107). Voir à ce sujet, l'étude comparée de Lévinas et Adorno que propose Orietta Ombrosi dans «L'umano ritrovato », 2010. 
soit le désastre comme pensée, ${ }^{3}$ l'impossibilité du sens comme ce qui désigne toute la tâche de la pensée.

Vis-à-vis de cet échec mais aussi de cette nouvelle tâche que la souffrance représente au regard de la pensée et en tant que la pensée, Lévinas semble avoir une position ambivalente. D'un côté, comme on vient de le voir, pour Lévinas la souffrance est inutile. Elle est, dans sa façon de se produire, cela même qui se refuse au sens et qui en produit l'échec. Pour cette raison, et non sans rappeler certains passages de «La généalogie de la morale » de Nietzsche, ${ }^{4}$ Lévinas s'oppose à l'usage disciplinaire et rationnel de la douleur par quoi la souffrance reviendrait à faire partie d'une économie, à délivrer une utilité. ${ }^{5}$ Sa critique de la théodicée comprend donc le refus de l'idée que la souffrance puisse rendre meilleur, qu'elle pourrait permettre d'expier un péché ${ }^{6}$ comme le dit Lévinas dans " La souffrance inutile » ou de " compenser une dette » pour reprendre cette fois-ci les mots de Nietzsche (GM, 76). De Nietzsche, Lévinas reprend d'ailleurs explicitement les mots lorsqu'il demande, à la façon d'une affirmation, « Le mot de Nietzsche sur la mort de Dieu ne prenait-il pas dans les camps d'extermination la signification d'un fait quasi empirique ?» (SI, 115). La disproportion exhibée à Auschwitz entre la souffrance et toute théodicée se passe du long chemin du généalogiste de la morale, elle impose silencieusement la vérité que formulera le Gai savoir concernant la mort de dieu. Mais d'un autre côté, l'œuvre de Lévinas où le thème de la souffrance est

3 La souffrance, décrite par Blanchot dans «L'écriture du désastre " comme « le subissement, le 'pas' du tout à fait passif en retrait par rapport à toute vue, tout connaître " est en fait l'objet d'un retournement, quant à ce qu'on appelle pensée. $\mathrm{Si}$, dans la mesure où elle est l'expérience d'un subir qui se dérobe au sujet, la souffrance est impensable (" en retrait par rapport à [...] tout connaître »), elle décrit en fait la pensée non en tant qu'acte réflexif mais en tant que « ce qui, dans la pensée, ne saurait se rendre présent, entrer en présence, encore moins se laisser représenter ou se constituer en fond pour un représentation " (p. 57). En d'autres termes, la passivité de la souffrance constitue un "passif de pensée " (ibid.), cela même qui, nous empêchant d'en finir, de nous abstraire, constitue la pensée. Voir Blanchot, 1980. pp. 11 et 57.

4 Dans « Généalogie de la morale », Nietzsche explique par exemple comment le sentiment de la faute naît de la souffrance infligée par le châtiment. La généalogie montre en ce sens en quoi ces catégories morales ne sont pas innées. Voir NIETZSCHE, F. « La généalogie de la morale » (dorénavant abrégé GM), Paris: GF, 2002, p. 93. Traduit en français par Eric Blondel, Ole Hansen-Love, Théo Leydebach, Pierre Pénisson.

5 Dans "La souffrance inutile ", Lévinas, non sans faire écho à certaines thématiques de "La généalogie de la morale ", évoque la " crainte du châtiment », les " sanctions » et la " répression » comme autant d'exemples d'une " téléologie politique » fondée sur la " santé admise comme fin suprême et ultime » de la société. Mais si la souffrance est par elle-même inutile, il n'y a pas de différence, suggère Lévinas, entre la « rationnelle administration de la douleur » et les " souffrances inutiles qui dérivent des fléaux naturels comme effets d'une perversion ontologique » (SI, pp. 112-3).

6 Lévinas qualifie ainsi la théodicée de " perspectives suprasensibles pour entrevoir dans la souffrance, essentiellement gratuite et absurde et apparemment arbitraire, une signification et un ordre » (ibid, p. 113). Comme le Nietzsche de la " Généalogie de la morale ", Lévinas critique l'économie de la souffrance et la lecture téléologique du péché originel : "Voilà la grande idée nécessaire à la paix intérieure des âmes dans notre monde éprouvé. Elle est appelée à faire comprendre les souffrances d'ici-bas. Celles-ci prendront un sens par référence à une faute originelle ou à la finitude congénitale de l'être humain » (ibid). 
originel et récurrent se déborde et se structure autour du problème du rapport entre la souffrance et Autrui. Or, tandis que, abordée phénoménologiquement, Lévinas décrit la souffrance comme cela même qui se refuse au sens, Lévinas affirme dans «Autrement qu'être » par exemple, que la souffrance pour Autrui inaugure la possibilité du sens. Pour Lévinas en effet, la possibilité du sens tient à ce qui structure le sujet " pour Autrui ». Or, « le pour-l'autre (ou le sens) va jusqu'au par-l'autre, jusqu'à souffrir par une écharde qui brûle la chair $[\ldots] » .^{7}$ Par ailleurs, toujours dans « La souffrance inutile », Lévinas fait de la « douleur d'autrui ", " le phénomène même de la souffrance dans son inutilité » (SI, 116). Tout se passe donc comme si c'était dans ce que Lévinas appelle 1' " ordre interhumain » (ibid.) que s'impose à la pensée cette vérité que la souffrance est ce qui se refuse au sens. Tandis que ma souffrance est inassumable et m'accable, celle d'autrui est injustifiable et me révolte. Ce renversement de perspective constitue-t-il une fuite par rapport à la critique et à l'échec de la théodicée que représentait jusqu'ici le thème de la souffrance ? Retrouvons-nous avec le thème de la souffrance pour Autrui le cas de figure longuement décrit dans " La généalogie de la morale » et dans « L'antéchrist » de Nietzsche d'une dénégation de la souffrance inhérente à la vie au profit de ce qui pourrait apparaître comme une économie de la compassion ${ }^{8}$ où la possibilité de souffrir pour Autrui viendrait redonner du sens à l'existence ? Ou bien dans la différence entre l'inassumable et l'injustifiable, Lévinas ne perçoit-il pas une issue à la complaisance que pourrait constituer la pensée de la souffrance, à l'immobilité dans laquelle pourrait confiner son non-sens constitutif, et au confort théorique que représente l'absence de sens quand ce dernier n'est que le négatif du sens ? Que veut dire Lévinas lorsqu'il affirme que penser la souffrance dans une perspective interhumaine restitue la souffrance « aux dimensions du sens »?

Afin de répondre à cette question, j'aimerais situer le motif de la souffrance pour Autrui chez Lévinas dans l'orbe de la critique nietzschéenne de la compassion et plus précisément de sa critique de la pitié. ${ }^{9}$ Pour Nietzsche en effet, du moins pour le Nietzsche de «L'antéchrist», « la pitié est la pratique

7 LEVINAS, E. «Autrement qu'être ou au-delà de l'essence » (dorénavant abrégé AE), Livre Poche, SD, p. 85.

8 Dans la « Généalogie de la morale », Nietzsche affirme que « ce qui excite en fait contre la souffrance, ce n'est pas la souffrance en soi, mais ce qu'elle a d'absurde » (GM, p. 79). Dans les mots de Lévinas, ce qui constitue la dolance de la souffrance, c'est son inutilité, c'est le fait que ce qu'elle produit c'est l'impossibilité du sens. Dans une optique généalogique, la compassion, la pitié, le fait de souffrir pour Autrui, constituent autant de calculs pour se soustraire à ce que la souffrance a d'absurde. Dans ce sens, le calcul chrétien consiste bien à redonner un sens à la souffrance en faisant d'elle un moyen pour l'obtention du salut.

9 Bien qu'il y ait chez Nietzsche une valorisation de la compassion (en particulier dans « Ainsi parlait Zarathoustra »), on ne distinguera pas ici pitié et compassion. Les traductions françaises de Nietzsche 
du nihilisme » (« Mitleiden ist die Praxis des Nihilismus »). ${ }^{10}$ Dans cette occurrence, Nietzsche pense à la dimension réactive du nihilisme, c'est-à-dire au nihilisme comme négation de la vie. La pitié étant l'amour pour le faible, pour « ce qui est mûr pour la disparition » (ibid.) comme dit Nietzsche, elle relèverait d'une dénégation de la vie qui est, au contraire, force affirmative, expansion, et même force de destruction (GM, p. 87). En situant le thème de la souffrance pour autrui dans l'orbe de la critique nietzschéenne de la compassion et dans celle du problème du nihilisme (entendu comme négation de la vie) on pourra préciser la façon dont celle-là renoue avec le sens ou déjoue ce qui relève encore de la dialectique entre sens et non-sens. Si la souffrance pour autrui relève bien du schéma nihiliste qu'analyse Nietzsche, elle s'incère alors dans cette mécanique qui consiste à chercher une rédemption au non-sens de la souffrance en trouvant un sens à la souffrance d'autrui. Ici, la vie, dans son aberration, serait niée au profit d'un calcul rédempteur. ${ }^{11}$ Mais si la souffrance pour Autrui telle que la décrit Lévinas échappe à ce calcul rédempteur, elle pourrait ouvrir de nouvelles lumières pour penser aussi bien le problème du sens que de la vie. Dans ce dernier cas, l'élaboration lévinassienne de la critique nietzschéenne de la compassion pourrait s'avérer continuer le projet nietzschéen, mais en le radicalisant.

\section{La lecture ambivalente de Nietzsche}

L'analyse que fait Lévinas du thème de la souffrance et plus précisément de son rôle dans la genèse du sujet marque tout à la fois un point de rencontre

traduisent indifféremment " mitleiden » (mit : avec : leiden : souffrir) par pitié ou compassion. Au sujet du rapport entre pitié et compassion, voir Frazer, 2006, pp. 49-78.

10 Nietzsche, 1967, p. 75. Traduit en français par Robert Rovini.

11 On pourrait opposer à cette lecture de la souffrance tragique décrite ici, dans l'orbe de Nietzsche et de Lévinas, l'idée que chez Nietzsche la souffrance a bien un degré d'utilité. De même que l'idéal ascétique correspond à un déni du corps, la souffrance permet de réaffirmer le corps et de devenir plus fort. Toutefois, en plus du fait qu'il faudrait à ce sujet distinguer souffrance et douleur, l'idée d'une utilité de la souffrance chez Nietzsche repose sur une lecture unilatérale de l'ensemble de l'œuvre de Nietzsche dans laquelle la vie serait le sens ultime de sa pensée. Le problème de ces lectures tient à ce qu'elles subsument cette pensée à un principe unificateur et risque même parfois de faire de la vie une nouvelle idole. Vis-à-vis de ces lectures, Barbara Stiegler dans son livre "Nietzsche et la critique de la chair. Dionysos, Ariane, le Christ » (2005), montre justement que la souffrance n'obéit pas à un schéma vitaliste. En mettant au centre de son analyse le " concept de Dionysos » (c'est-à-dire du dieu souffrant), Stiegler montre que pour Nieztsche, la souffrance est en excès sur toute utilité. Stiegler décrit alors la question de la souffrance chez Nietzsche dans des termes qui sont presque identiques à ceux qu'emploie Lévinas dans " La souffrance inutile » : "La souffrance souffre [...] du fait que ce qui se donne se donne en trop " (Stiegler, 2005, p. 80), et " cette 'sagesse du souffrir', c'est d'abord la reconnaissance de la passivité fondamentale qui borne l'activité représentative " (ibid., p. 78). Au sujet d'une étude comparée de la question de la souffrance chez Nietzsche et Lévinas, et de l'utilité de la douleur chez Nietzsche, voir la très stimulante analyse de Pierre Zaouï dans « La traversée des catastrophes. Philosophie pour le meilleur et pour le pire » (2010). Au sujet d'une lecture non-utilitaire de la souffrance chez Nietzsche et de ses rapports avec la pensée de Lévinas, voir : Edgar, 2007. 
et un point de divergence vis-à-vis de la pensée de Nietzsche. D'un côté, et en cela Lévinas est proche du Nietzsche de "La naissance de la tragédie », Lévinas fait de la souffrance le point de départ de l'annonce d'un tournant dans ce qu'il appelle la « culture européenne $»,{ }^{12}$ c'est-à-dire d'une culture et d'une tradition fondée sur le primat de l'idée et sur la dénégation du corps. D'un autre côté, tandis que le même Nietzsche de "La naissance de la tragédie » pense la souffrance en termes esthétiques dans le jeu qui oppose le dionysiaque de l'apollinien, Lévinas pense que c'est l'éthique (celle qui se produit comme « souffrance pour Autrui ») qui permet de penser la souffrance dans toute sa nudité (c'est-à-dire dans la radicalité de l'épreuve du non-sens). Essayons tout d'abord de comprendre comment et pourquoi se produit cette divergence au lieu même où ces deux pensées se rencontrent. Pour cela j'aimerais aborder brièvement le texte de Lévinas "Quelques réflexions sur la philosophie de l'hitlérisme » écrit en 1934. Bien qu'il s'agisse d'un texte de jeunesse de Lévinas - il est intéressant d'observer qu'il s'agit d'un texte d'avant guerre - , il peut être considéré comme un prélude à bien des inquiétudes de l'œuvre à venir de Lévinas. ${ }^{13}$

Dans ce texte « Quelques réflexions sur la philosophie de l'hitlérisme », Lévinas affirme que le marxisme, dans le poids qu'il confère au matérialisme, " brise la ligne harmonieuse » " de la culture européenne » (QQPH, p. 14). Dès ses premiers textes, c'est donc la rupture avec le primat de l'esprit (caractéristique, selon Lévinas, de la " culture européenne ») qui intéresse Lévinas. Une des idées maîtresses de ce texte est d'ailleurs que l'hitlérisme, en tant que politique fondée sur l'idée de race, est le résultat de la dénégation du corps qui caractérise la culture européenne. Ces deux éléments indiquent que la pensée de Lévinas se constitue dans le souci du corps et de la matérialité, dans la nécessité de sortir d'un certain idéalisme fondé sur la négation (ou l'ignorance du corps) - et dans ce sens dans la nécessité de suivre Marx au lieu de cette brisure de « la ligne harmonieuse de la culture européenne »mais aussi dans l'inquiétude de ce que peut représenter le primat du corps au regard du politique. Si l'on suit Marx, le matérialisme répond à une exigence de penser concrètement la justice, de ne plus se suffire d'une justice qui serait pensée en idée ; mais si l'on suit le diagnostique qui concerne l'hitlérisme, le problème du corps réside aussi dans l'affirmation brute et aveugle de la

12 LEVINAS, E. "Quelques réflexions sur la philosophie de l'hitlérisme " (dorénavant abrégé QQPH), Paris : Rivages, 1997.

13 A ce sujet, voir l'essai de Miguel Abensour dans "Quelques réflexions sur la philosophie de l'hitlérisme ", op.cit. 
force. Or, dans ce texte de « jeunesse », Nietzsche est présent au moins à deux moments, une fois en sourdine et une seconde fois de façon explicite. Le passage par ces deux occurrences va nous permettre de comprendre pourquoi la compréhension tragique de la souffrance va être redoublée par sa compréhension éthique.

Dans le premier cas, Nietzsche est présent lorsque Lévinas cherche à penser la naissance du sujet à rebours de tout dualisme. A ce propos, Lévinas laisse entendre déjà dans ce texte que la genèse de la conscience dérive du corps (idée qui sera développée de façon plus détaillée par la suite, en particulier dans « De l'existence à l'existant»). Jamais nommé au début du texte, on peut cependant dire que Nietzsche est présent dans le renversement des rapports entre l'âme et le corps. Comme pour Nietzsche, pour Lévinas le corps n'est pas l'étranger dans lequel l'âme déchoit, comme si elle lui préexistait. Le corps au contraire, est constitutif de l'individualité. ${ }^{14}$ Le corps « n'est pas seulement l'éternel étranger » et il n'est pas seulement " plus familier » écrit Lévinas dans « Quelques réflexions sur la philosophie de l'hitlérisme ». Avant d'être étranger ou proche, le corps est ce dans quoi se forge le « moi » ou ce que, dans ce texte de 34, Lévinas appelle le « sentiment d'identité »(QQPH, p. 16). Or, à cet égard, le thème de la douleur sert à exemplifier le fait de cette union : « Et dans l'impasse de la douleur physique, demande Lévinas à la façon d'une constatation, le malade n'éprouve-t-il pas la simplicité indivisible de son être quand il se retourne sur son lit de souffrance pour trouver la position de la paix ? » (ibid., p. 17). Dans la douleur en effet, le « moi » ne peut échapper à soi, il ne peut s'abstraire de soi. La douleur a alors un rôle génétique. C'est dans la souffrance que l'âme se noue au corps, que se produit l'indivision de l'individu, c'est-à-dire le moi. La douleur permet donc de décrire la genèse de l'individu mais d'un individu dont la naissance est suspendue au caractère tragique de la souffrance.

Mais Nietzsche est présent une seconde fois dans ce texte, et cette foisci, de façon explicite. Tandis que Lévinas renverse - pour le dire de façon caricaturale - les fondements de la culture européenne, ou du moins de ce que Lévinas caractérise comme tel, il montre aussi que c'est de ce renversement que

14 Dans «L'un pour l'autre. Lévinas et la signification » (2008), Didier Franck a analysé le problème des rapports entre l'âme et le corps chez Lévinas tout en indiquant que sur ce sujet, Lévinas n'atteint pas la radicalité du primat du corps tel que Nietzsche l'aurait pensé. Pour Franck en effet, l'incarnation dépendrait chez Lévinas de la subjectivité, tandis que pour Nietzsche, à l'inverse, la subjectivité et la conscience dépendent du corps. Or, ce que nous allons montrer par la suite est que chez Lévinas la subjectivité dérive de la sensibilité et en particulier de la façon dont la rencontre éthique est constitutive de la sensibilité. Par ailleurs, nous proposons de suivre Barbara Stiegler pour qui le corps chez Nietzsche ne se réduit pas à un jeu de force mais doit être appréhendé comme chair (corps incarné). Voir à ce sujet la note 25. 
se nourrit la « philosophie de l'hitlérisme ». D'une certaine façon, l'hitlérisme serait une pensée (une « philosophie ») en cela qu'il se nourrit de ce que la culture européenne a ignoré (ou nié) pour se constituer comme telle, à savoir le corps. Mais l'hitlérisme est une pensée qui ne se pense pas justement. Le corps qu'il affirme - le corps aryen - est seulement un corps qu'il impose. De là le souci qu'explicitera Lévinas au regard de la pensée de Nietzsche. Pour Lévinas, Nietzsche ne s'est pas contenté de penser le primat du corps, il a pensé le corps comme force qui s'impose, et cela parce que le corps, comme succédané de la vérité, devait s'universaliser pour prendre la place de la vérité. Pour illustrer cette idée, Lévinas prend l'exemple de Zarathoustra qui ne cherche pas son propre dépassement sans revenir vers les hommes. Selon Lévinas, «Zarathoustra ne se contente pas de sa transfiguration, il descend de sa montagne et apporte un évangile » (ibid., p. 22). Ainsi, pour Lévinas, bien qu'en porte à faux avec la vérité, Nietzsche aurait quand même eu besoin, pour affirmer sa vérité, d'universalité. Dans son renversement du platonisme, Nietzsche aurait cherché à penser le corps dans la même dimension selon laquelle Platon pensait l'idée. Si la vérité de l'idée est universelle, le corps doit également relever de l'universel. Mais, demande Lévinas, " comment l'universalité est-elle compatible avec le racisme » (ibid.) ? Le primat du corps ne vise-t-il pas en effet à affirmer les sensations et donc un point de vue subjectif ? Comment le corps - et plus précisément le corps pensé en termes de race, c'est-à-dire de façon éminemment particulière - peut-il prétendre au statut de vérité universelle ? A cette question, Lévinas répond « par la guerre et la conquête » (ibid., p. 23). Ce n'est que par la force que la vérité du corps peut acquérir un statut universel ; cette dernière ne peut être que forcée, imposée. Tandis que l'idée exige la réflexion, le corps se manifeste par l'expansion ; tandis que l'universalité de l'idée se propage par la persuasion, la forme d'universalisation du corps est la force. Et à Lévinas de conclure : « La volonté de puissance nietzschéenne que l'Allemagne moderne est en train de redécouvrir et de glorifier, n'est pas seulement un nouvel idéal. C'est un idéal qui apporte en même temps avec lui sa propre forme d'universalisation : la guerre et la conquête » (ibid.).

\section{L'ambivalence de la douleur}

Indépendamment de la question de savoir si Lévinas se trompe ou non vis-à-vis de Nietzsche, ce qu'il importe est de comprendre que cette position ambivalente vis-à-vis du corps (et donc vis-à-vis de Nietzsche) tient à l'ambivalence du phénomène de la souffrance. Cette ambivalence nous 
reconduira par la suite à la différence entre une conception tragique et une conception éthique de la souffrance. En effet, cette ambivalence se noue autour du problème de l'individuation et de l'anonymat de la souffrance. D'un côté, comme on l'a vu, Lévinas montre que la souffrance joue un rôle crucial dans l'individuation du sujet ; mais d'un autre côté, la souffrance est aussi ce qui désindividue le sujet, ce par quoi, acculé à soi par la douleur, le sujet n'est plus sujet, il n'est plus que douleur, plus que le cri et l'impossibilité d'être soi qui se produit dans la douleur. ${ }^{15}$

On retrouve la description de cette ambivalence dans les «Quelques réflexions sur la philosophie de l'hitlérisme ». Comme on l'a vu, la douleur produit l'indivision; mais la douleur est aussi ce qui « rive à soi ». Or, être rivé à soi c'est perdre ce qui, pour la culture européenne, fait la grandeur du soi, à savoir la liberté. Dans « Quelques réflexions sur la philosophie de l'hitlérisme », Lévinas décrit déjà cet être rivé comme un enchaînement, et donc comme un emprisonnement, et finalement comme une aliénation du soi. Ainsi comprendt-on que la philosophie de l'hitlérisme, qui est une philosophie de la force, est une philosophie de la non-liberté qui renonce finalement à tout ce qui faisait la valeur de l'humain. Mais plus intéressant encore est de constater que cette non-liberté résulte de l'identité, du type d'indivision que produit la souffrance. Si la douleur produit l'indivision, cette indivision, en tant qu'identité à soi, n'est qu'une forme d'emmurement, d'engloutissement en soi. Ainsi d'une part la souffrance ${ }^{16}$ est-elle aliénante puisque le soi n'y est pas libre, mais cette aliénation répond à la structure de l'identité. Elle est celle d'un emmurement

15 Blanchot a très bien décrit cette perte d'individualité qui se produit dans la douleur dans L'entretien infini, en particulier dans le chapitre «L'espèce humaine ». A ce sujet, voir aussi le chapitre « Le pendule équivoque » dans «La traversée des catastrophes. Philosophie pour le meilleur et pour le pire » (op. cit.), de Pierre Zaouï.

16 Lévinas ne fait pas systématiquement la différence entre la souffrance et la douleur, qui se co-impliquent d'ailleurs mutuellement. En dépit du fait que l'un et l'autre ne sont pas simplement dissociables, on peut toutefois dire que la douleur relève d'une sensation localisable et la souffrance d'un état ou d'une condition. Dans « La souffrance inutile », Lévinas décrit la souffrance comme une donnée de la conscience (il ne l'a dissocie donc pas de la douleur qui, en tant que sensation, relève bien du vécu), mais il précise que ce qui caractérise une telle donnée est la façon dont elle se présente : « Mais dans ce contenu même elle est un malgré la conscience : l'inassumable ». Pensée de cette façon, la souffrance est donc moins qu'un état. En tant que «l'inassumable », elle est ce qui ne se laisse pas stabiliser, ce qui déporte le sujet hors de tout confort. C'est ce qu'on peut entendre dans la formule « incondition d'étranger ». Au sujet de la difficulté de distinguer souffrance et douleur, voir également Paul Ricoeur, « La souffrance n'est pas la douleur ", in Autrement, Souffrances (1994) repris dans Souffrance et douleur. Autour de Paul Ricoeur (2013, dir. Claire Marin et Nathalie Zaccai-Reyners) et Didier Franck, « Nietzsche et l'ombre de Dieu » (1998). A partir de sa lecture de la question du corps chez Nietzsche, Didier Frank s'oppose à l'idée que la douleur (à la différence de la souffrance) serait simplement localisable. Pour Didier Franck en effet, celle-ci atteste d'un déséquilibre dans l'ensemble du corps et se manifeste déjà comme évaluation (ibid., p. 205). Dans cette lecture, c'est toutefois uniquement la dimension active de la douleur qui est prise en compte et non le souffrir comme impouvoir. 
en soi. Le texte de 1934 (et ce sera le cas de tous les textes qui suivent) montre bien que la souffrance est un phénomène ambivalent et tire les conséquences de cette ambivalence. Comme le remarque à juste titre Rodolphe Calin dans ses analyses des textes plus tardifs de Lévinas ${ }^{17}$ (analyses qu'on pourrait appliquer à ce texte de jeunesse), la souffrance individue et déjà aliène, force le soi, mais un soi qui n'est déjà pas soi. La conséquence de cette ambivalence réside dans la nécessité mais aussi dans le danger d'une pensée qui affirme le primat du corps, comme celle de Nietzsche. Ce texte de 1934 indique donc qu'il est d'un côté nécessaire de penser le corps pour sortir de ce que Lévinas appelle parfois « l'hypocrisie de sermon $\|^{18}$ qui n'est rien d'autre que l'hypocrisie de l'idéalisme et que Nietzsche appelle " optimisme théorique ${ }^{19}$; mais que, d'un autre côté, abandonnée à elle-même, la pensée du corps est une pensée aveugle, une pensée sans moi, où ne s'affirme plus alors qu'une force brutale, et qui ferait l'essentiel de la " philosophie de l'hitlérisme ». Nécessaire philosophiquement, puisque le corps a un rôle génétique dans la naissance du moi, la pensée du corps est aussi éminemment dangereuse politiquement. Mais Nietzsche n'a-t-il pas perçu cette ambivalence de la souffrance?

Dans un sens, cette ambivalence de la souffrance est incarnée par les pôles antagonistes qui constituent la « Naissance de la tragédie » de Nietzsche, à savoir l'apollinien et le dionysiaque. Pour Nietzsche en effet, l'apollinien relève d'un principe d'individuation où la souffrance se limite à individuer. En revanche, le dionysiaque traduit ce qui dans la souffrance est anonyme. Le dionysiaque correspond à la souffrance de la vie et non à celle de l'individu. Il exprime la souffrance en tant qu'elle anéantit l'individu et le ramène au caractère anonyme de la vie. Or, pour Lévinas, cette vision de la souffrance n'est pas une vision. Elle peut bien excéder les limites de l'individu dans l'expression d'un regard impersonnel ; mais dans cet anonymat, elle ne peut plus rien voir parce qu'elle ne sort plus de soi. Tragique, le dionysiaque le serait en tant qu'il se complait dans l'absence de liberté, dans une forme

17 Rodolphe Calin perçoit très bien ce paradoxe dans "Lévinas et l'exception de soi ». A différence de Jean-Luc Marion qui, dans « De surcroît », insiste sur le fait que la souffrance coïncide avec le moment d'individuation du moi, Rodolphe Calin, insistant sur le fait que cette individuation est sans sujet, parle plutôt d'une « souffrance (dés)individualisante ». Voir Calin (2005, pp. 85-122) et Marion (2001). A ce débat, on pourrait ajouter qu'il ne s'agit pas d'opposer deux conceptions différentes de la souffrance mais deux modalités différentes du souffrir. Comme on va le voir, la souffrance éthique, le « souffrir pour Autrui » qui selon Lévinas structure la subjectivité, excède le caractère désindividualisant de la souffrance tragique sans cependant jamais s'en préserver complètement. Marion a raison de dire que « Le moi ne se fixe que quand il prend chair » (ibid., p. 114), mais il faudrait ajouter que c'est parce que sa chair n'est jamais indemne d'Autrui que le moi ne se dissout pas dans l'impuissance de sa souffrance, dans l'anonymat de sa chair.

18 Lévinas (1993, p. 69).

19 Nietzsche (1986, p. 102). 
d'emprisonnement qui ne permet pas de liberté réelle. En quoi cependant la souffrance éthique, la souffrance pour Autrui, permettrait-elle de « dépasser » cet emmurement tragique, sans toutefois céder au subterfuge qui consisterait à retrouver un sens à la souffrance, à " souffrir pour » afin de retrouver une utilité à la souffrance et se délester de son absurdité ?

\section{Souffrance éthique et liberté}

Le thème de la souffrance pour Autrui qui ne sera d'ailleurs pas un thème parmi d'autre mais bien, comme on l'a vu, ce qui inaugure la possibilité du sens et ce à quoi tient la structure de la subjectivité, n'a pas seulement pour Lévinas un enjeu éthique, il ouvre sur ce que Lévinas appelle la « difficile liberté ». En effet, tandis que le texte de 1934 invitait à la nécessité de penser le primat du corps mais tout en laissant voir les dangers d'une telle pensée, le thème de la souffrance pour Autrui va rendre possible une nouvelle articulation entre les thèmes du corps et de la liberté. Par là, si du moins on considère que la liberté est bien un problème politique, l'enjeu du thème de la souffrance pour Autrui n'est pas seulement éthique. Voyons d'une part comment la souffrance pour Autrui permet de sortir de l'impasse sur laquelle se termine le texte de 1934 (mais qui marque tout un programme à venir) et d'autre part comment cela se raccorde à notre problème initial, à savoir le rapport entre la souffrance et le nihilisme.

Tandis que, comme on l'a vu, la souffrance a un rôle génétique, la souffrance pour Autrui poursuit ce même rôle mais permet de sortir de la contradiction dans laquelle se maintenait enfermé l'individu rendu indivisible par la douleur. En effet, la souffrance pour Autrui permet à Lévinas de décrire non la genèse du moi mais celle de la sensibilité. C'est alors à partir de cette nouvelle compréhension de la sensibilité que Lévinas pourra penser une subjectivité qui ne perd rien de son unicité, qui ne reste pas emmurée dans l'individualité.

C'est dans « Autrement qu'être » que se produit cette articulation entre sensibilité et subjectivité. ${ }^{20}$ Tandis que dans "Quelques réflexions sur la philosophie de l'hitlérisme », Lévinas montre que le moi n'est pas préalable au corps, dans «Autrement qu'être », Lévinas montre que la sensibilité n'est pas préalable à la rencontre : elle en dérive. Ainsi, dans le chapitre « vulnérabilité et contact » de la section « Sensibilité et proximité » Lévinas affirme-t-il « la 
sensibilité est exposition à l'autre » (AE, p. 120). Cette nouvelle conception de la sensibilité s'explique par le fait que, tandis que traditionnellement la sensibilité est définie comme perception, c'est-à-dire comme une forme de compréhension de l'objet, dans la rencontre (comprise comme « face-à-face ») avec Autrui, la sensibilité est débordée par son objet. Ainsi, «La sensibilité est affection par le non-phénomène, une mise en cause par l'altérité de l'autre » (ibid., p. 121). Dans le face-à-face, la sensibilité n'est pas dans un rapport de prise ; débordée par l'Autre, elle est offerte. Lévinas peut donc parler de sensibilité comme « exposition à l'autre 》(ibid.,p. 120), comme «vulnérabilité» (ibid.), comme « avoir-été-offert-sans-retenu » (ibid.) parce que ce n'est pas la sensibilité qui définit l'objet mais la nature de la rencontre qui produit la sensibilité. Or, que la sensibilité soit « exposition à l'autre » signifie que c'est la souffrance pour Autrui, cet « avoir-été-offert-sans-retenu » qui fait la sensibilité. Mais ici, à la différence d'un corps qui individue mais enferme le sujet, la souffrance pour Autrui forge la sensibilité dans ce qui ouvre le sujet, et plus précisément dans ce qui l'expose, dans ce qui l'offre à Autrui. Dans le faceà-face éthique, la subjectivité, structurée comme « pour Autrui » («Autre dans le Même » écrit souvent Lévinas) n'est pas seulement rendue vulnérable par Autrui, elle souffre de tous les autres qu'elle porte en tant que, paradoxalement, elle leur est offerte. Dans " Autrement qu'être », Lévinas parvient donc à redonner au corps toute sa primauté sans abandonner la subjectivité au risque de l'enfermement et de la brutalité. La souffrance pour Autrui est en effet ce qui permet de comprendre à nouveau frais l'articulation entre sensibilité et subjectivité. Dans la sensibilité conçue comme « exposition à l'autre », le moi n'est pas emmuré en lui-même et ainsi annulé dans le corps qui l'individue ; il est rendu unique par ce qui l'expose et donc l'ouvre, brise le sceau de son enfermement. Mais on comprend aussi pourquoi cette nouvelle articulation entre sensibilité et subjectivité débouche sur une " difficile liberté ». Ouvert dans la souffrance pour Autrui, la subjectivité sort de son emmurement. En cela, le « pour Autrui » de la souffrance est ce qui libère le moi. Mais ce « pour» est aussi ce par quoi la subjectivité porte toujours déjà l'autre en soi. Dans cette nouvelle genèse de la subjectivité, « je suis noué aux autres avant d'être noué à mon corps » (ibid., p. 123) comme écrit Lévinas. L'unicité du sujet se produit dans une sensibilité qui est un « avoir-été-offert-sans-retenu » qui est un porter l'autre, dans ce que Lévinas appelle un « corps maternel » (ibid.). La liberté du sujet n'est donc possible que comme responsabilité, comme ce porter l'autre dans ce qui m'offre à l'autre, comme « difficile liberté ». Il ne s'agit pas d'une liberté fondée dans une compréhension idéale de l'esprit où le moi revient toujours au même mais comme l'écrit Lévinas dans « Totalité 
et infini » d'une « liberté investie $»^{21}$ où la subjectivité a toujours déjà été mise en jeu par Autrui. Si le moi identique à soi est finalement enchaîné à soi, la " difficile liberté » doit être comprise non comme pouvoir mais comme ce qui affranchit le soi de ses chaînes, c'est-à-dire comme sortie. Cette nouvelle articulation entre sensibilité et subjectivité permet donc de déjouer aussi bien 1' « hypocrisie de sermon » des idéalismes que l'aveuglement et la brutalité d'une pensée où le corps s'affirme seul, indépendamment du mode selon lequel l'épreuve de l'altérité constitue la sensibilité.

Elle permet également de comprendre comment Lévinas élabore à nouveau frais la question du sens. Tandis que, comme on l'a signalé en introduction, d'un côté, la souffrance est, par elle-même, par sa façon de se produire, inutile, absurde, tragique, d'un autre côté, la souffrance pour Autrui constitue l'ouverture du sens. Mais ce qu'il nous faut montrer à présent, c'est, d'une part, en quoi la souffrance éthique nous permet de comprendre la question de la genèse du sens chez Lévinas, et de quelle façon elle ne constitue pas simplement une relève de la souffrance tragique, c'est-à-dire en quoi elle demeure « inutile ». Cette articulation entre souffrance éthique et souffrance tragique est au cœur d' " Autrement qu'être » où on lit par exemple : « Le pour-l'autre (ou le sens) va jusqu'au par l'autre, jusqu'à souffrir par une écharde qui brûle la chair, mais pour rien » (déjà cité, $\mathrm{AE}, \mathrm{p} .85$, je souligne). Comment comprendre cette élaboration de la question du sens qui ne retire rien au tragique de la souffrance ? En pensant la question du sens à partir de la « souffrance pour Autrui », Lévinas pense le sens comme ce qui ouvre le sujet hors de son enfermement, non comme fin. Le sens est l'ouverture du sujet hors de son monde clôt. Il se tient dans ce qui fissure le sujet et le structure « pour Autrui ». Ce qui importe ici est que ce qui ouvre le sujet ne vient pas de lui (autrement, il demeurerait fermé dans son ouverture). Pour Lévinas,

21 A ce sujet, voir Lévinas, Totalité et Infini, « L'investiture de la liberté ou la critique », pp. 83-90. On peut être surpris de cette idée que l'articulation entre sensibilité et rencontre éthique donne lieu à une élaboration de la question de la liberté. La rencontre éthique dans «Autrement qu'être ou au-delà de l'essence » est souvent lue dans le sens contraire, à savoir, comme l'avènement d'une perte de liberté. Dans le face-àface, le moi est bien « otage de l'autre ». Toutefois, si ces analyses sont justes et même fidèles à certaines formules de Lévinas (par exemple, « être responsable au-delà de sa liberté », AE, p. 194), il ne s'agit pas pour Lévinas de substituer la responsabilité à la liberté (c'est-à-dire l'éthique à la politique), mais de décrire une situation antérieure à l'autonomie du sujet et d'où sa liberté reçoit un sens positif. Ce qui est alors particulièrement intéressant est que cette antériorité, bien que décrivant une situation de captivité (être otage de l'autre) ne fait qu'accroître la liberté en libérant la liberté de ce qui l'enchaîne. II s'agit pour Lévinas de montrer que la liberté n'est pas une condition de l'humanité mais ce qui n'est rendu possible qu'à partir de l'éthique : «Si des termes éthiques surgissent dans notre discours, avant ceux de la liberté et de la non-liberté, c'est que, avant la bipolarité du bien et du mal présentés au choix, le sujet se trouve commis avec le Bien dans la passivité même du supporter. La distinction du libre et du non-libre ne serait pas l'ultime distinction entre humanité et inhumanité [...] » (ibid.). 
sans cette ouverture qui vient d'Autrui, rien ne viendrait à signifier, tout reviendrait au Même, c'est-à-dire au même enchaînement où le moi s'annule (et donc la liberté). Pour la même raison, si la souffrance n'était pas « pour rien », l'ouverture ne serait pas désintéressée. Elle ferait de nouveau le jeu du « Même " dans lequel le moi se retrouverait à nouveau enchaîné à lui-même. Comme l'écrit Lévinas : « La signification comme l'un-pour-l'autre, sans assomption de l'autre par l'un, dans la passivité, suppose la possibilité du nonsens pur envahissant et menaçant la signification. Sans cette folie aux confins de la raison, l'un se ressaisirait et, au cœur de sa passion, recommencerait l'essence » (ibid.). Tandis que la souffrance, dans son inutilité, dissous le sujet, l'anéantit, la " souffrance pour Autrui » relève d'un éveil. Cela ne veut pas dire que cette souffrance est sensée, mais au contraire que le caractère insensé de la souffrance d'Autrui est ce qui me meut hors de moi, ce qui m'éveille, ce qui m'interdit le repos dans l'absurde de la souffrance. Dans ce sens, non seulement la souffrance éthique n'est pas la relève de la souffrance tragique, mais on peut même penser qu'elle est la seule exposition au caractère tragique (inutile) de la souffrance. En effet, dans la souffrance tragique, je peux encore me complaire dans le non-sens de la souffrance - non-sens qui devient alors un sens car il fait encore le jeu du sens. Pour Lévinas en revanche, le « pour Autrui » est sens au sens où il dérange toute complaisance dans un sens acquis comme dans un non-sens qui viendrait se constituer encore en sens, en nouvel ordre des choses. Parce que la subjectivité se produit dans une sensibilité qui est vulnérabilité, qui est offerte, mais qui est offerte " pour rien » comme le précise Lévinas, le sens ne relève plus d'aucune économie, ni de celle d'un sens qui relèverait d'une théodicée ou d'une téléologie, ni de celle du non-sens conçu comme simple revers du sens et qui, conçu ainsi, relève encore d'une position, sinon confortable, du moins immobile.

On voit ainsi en quoi la dimension éthique de la souffrance permet d'excéder son expression tragique ou esthétique sans rien perdre de son non-sens, de son inutilité, de son " pour rien », c'est-à-dire sans céder à la tentation de la théodicée. En pensant que la souffrance pour Autrui inaugure le problème du sens, Lévinas loge bien, suivant une ligne qu'on pourrait peut-être encore appeler nietzschéenne, la question de la signification dans celle de la matérialité. C'est en effet, comme on l'a vu, la sensibilité pensée comme exposition, comme « avoir-été-offert-sans-retenu » qui permet de penser que le sens excède toute forme d'économie. Mais à la différence de Nietzsche, du moins du Nietzsche de «La naissance de la tragédie », pour Lévinas, c'est l'éthique qui est la véritable épreuve du « pour rien » de la souffrance. Le « pour l'autre », qui est aussi un «par l'autre », la subjectivité 
« exposée à tous les vents », le sujet mis hors de soi par Autrui mais rendue unique par Autrui, porte Autrui, s'offre à Autrui et souffre pour lui dans la mesure où cette souffrance est « pour rien ». Or, ce " pour rien » ne relève plus de la complaisance théorique où sens et non sens s'opposent comme deux catégories logiques. Tant que l'inutilité de la souffrance pouvait s'oublier dans l'anonymat des masques de Dionysos, elle pouvait encore se complaire dans cet anonymat, et, dans cette conformité à soi, dans cette immanence, accepter sa brutalité inhérente. Dans ce sens, brutale, c'est-à-dire aveugle, la « souffrance inutile » n'est plus vraiment une épreuve du non-sens. Dans son immanence, il n'y a plus rien qui puisse prendre le sens du non-sens. En revanche, le « pour rien » de la « souffrance pour Autrui » relève toujours du refus que la souffrance d'Autrui provoque. C'est un refus du non-sens de la souffrance, mais qui ne procède d'aucun sens préalable (puisqu'il constitue la possibilité du sens) et qui demeure « pour rien».

\section{Au-delà de tout nihilisme : la vie créée}

Revenons à la question du rapport entre l'éthique de Lévinas et plus précisément la souffrance pour Autrui et le problème du nihilisme.

Pour Nietzsche comme je l'ai dit, " la pitié est la pratique du nihilisme » en cela qu'en se vouant au faible, à ce qui « est mûr pour la disparition ", elle nie la vie dans son caractère affirmatif, expansif, destructeur. Par ailleurs, Nietzsche observe dans la " Généalogie de la morale » que cette négation de la vie prend aussi la forme d'une négation de la souffrance, de ce que la souffrance a d'absurde comme dit Nietzsche. La morale est justement la tentative de refuser cet absurde en faisant de la souffrance « une compensation de la dette », c'est-à-dire en justifiant la souffrance au sein d'une économie où elle acquiert un rôle rédempteur.

On vient de voir du moins partiellement en quoi l'éthique de Lévinas échappe à ce schéma. La souffrance pour Autrui est une souffrance pour rien. Le " pour » du pour Autrui n'est pas une fin mais le refus de toute immobilisation dans le non-sens, immobilisation qui pourrait à son tours finir par constituer un sens. Mais il y a une deuxième amorce de réponse qui nous conduit à penser que non seulement la pensée lévinassienne ne tombe pas sous le coup de la critique nietzschéenne, mais conduit plutôt à radicaliser sa pensée.

Tout d'abord, il faut rappeler que, de façon évidente, le « souffrir pour Autrui » de Lévinas décrit tout le contraire qu'un rapport de pitié. Pour Lévinas Autrui est celui qui se présente de face, de ce fait, il se présente toujours par surprise, indépendamment de l'initiative d'un sujet. Le « face-à-face » est ce 
qui élève le regard et non ce qui l'abaisse. ${ }^{22}$ « Souffrir pour Autrui » ne relève donc pas d'un rapport de pitié entendu comme rapport de condescendance. La souffrance pour Autrui, on l'a vu, relève d'une dépense, non d'une économie par quoi le sujet se préserve - dans sa supériorité ou dans sa volonté de néant. Or, cette dépense rejoint la critique nietzschéenne d'une vie qui ne cherche que sa préservation. ${ }^{23}$

En effet, pensé en termes d'élévation, le duo éthique nous permet de revenir sur ce qui est au cœur de l'argumentation nietzschéenne : le problème de la vie. Ce qui est intéressant, est que, déjouant toute possible pitié - Autrui étant bien plutôt, à même sa fragilité, le maître (celui qui élève mon regard) -, la souffrance éthique est ce qui, à rebours de tout nihilisme (entendu comme négation de la vie), permet de redéfinir la vie, et même, d'en décrire à nouveau frais la généalogie. Tandis que pour Nietzsche la vie est ce qui se désire elle-même, ${ }^{24}$ et qui, par là, se déborde sans cesse, pour Lévinas, la vie procède du rapport à Autrui. Cette question est particulièrement développée dans "Autrement qu'être » où Lévinas décrit le rapport à Autrui en termes d'inspiration. La subjectivité décrite comme "Autre dans le Même » est littéralement animée ou inspirée par Autrui si bien que son ouverture coïncide avec son animation, c'est-à-dire avec le principe de sa vitalité. Comme l'affirme Alphonso Lingis dans sa préface à l'édition anglaise d'« Autrement qu'être », avec Lévinas, ce qui caractérise l'ouverture de la subjectivité n'est pas le fait d'ex-sister ou d'être-le-là (en cela, la subjectivité n'est pas Dasein) ; son ouverture est sa dénucléation, ce qui la perturbe dans son essence ou dans son repos et l'offre à Autrui. ${ }^{25}$ D'une façon tout à fait remarquable,

22 Dans «Totalité et Infini », Lévinas parle par exemple d'une « courbure l'espace » afin de qualifier la relation entre « être humains », où « Autrui se place plus haut que moi » (TI, p. 323).

23 Dans « Lévinas, the philosophy of suffering, and the ethics of compassion », Richard White remarque que l'usage du mot compassion est peu fréquent dans l'œuvre de Lévinas et il ajoute que la souffrance éthique (qu'il décrit, en reprenant les mots de Lévinas, comme " souffrance de la souffrance d'Autrui ") n'est au fond pas un rapport de compassion mais de devoir. S'il est vrai que les occurrences du mot compassion dans l'œuvre de Lévinas sont rares (mais non pas inexistantes), il ne s'agit pourtant pas pour Lévinas de remplacer la compassion (que White rapporte à l'amour) par le devoir. En fait, que ma sensibilité ne soit pas indemne de la souffrance d'Autrui, que je ne puisse souffrir sans souffrir aussi pour Autrui, cela ne définit pas un « devoir » au sens moral, cela m'élève plutôt au-delà de la compassion entendue comme pitié (où ma compassion me placerait dans un rapport de supériorité vis-à-vis d'Autrui). L'éthique ne se substitue donc pas à la compassion mais l'élève au-delà de l'égoïsme. La souffrance d'Autrui me précède ; elle est en cela non seulement ce qui m'ouvre au-delà des limites de mon moi, mais aussi ce qui m'appelle à excéder toutes formes d'économies : celles impliquées par la compassion comme celles impliquées par le devoir. Voir White (2012).

24 C'est la définition que Nietzsche donne de la vie dans la seconde "Considérations inactuelles » : " C'est la vie, la vie seule, cette puissance obscure qui pousse et qui est insatiable à se désirer elle-même », trad. Henri Albert, édition électronique, La gaya scienzia, Janvier 2012, p. 32.

25 En décrivant l'ouverture à l'air comme première par rapport à l'ouverture qui caractérise le Dasein, Alphonso Lingis insiste sur la rupture de Lévinas par rapport à tout intellectualisme. L'existence heideggérienne est 
Lévinas décrit l'animation de la subjectivité comme première par rapport à l'inspiration. Tout se passe comme si, avant le besoin d'air qui caractérise la vie, le sujet recevait la vie d'Autrui. Or, cela ne signifie pas que la vie dépend d'un créateur (le geste généalogique de Lévinas conduirait ainsi à revenir à une origine), mais que la subjectivité n'étant jamais indemne de l'autre, nous ne sommes plus en mesure d'assigner une primauté quelconque, pas même à la vie. Pour Lévinas, parce que la subjectivité naît d'avoir toujours déjà été affectée par Autrui, celle-ci peut être appelée " créature ». La notion de créature réfère au fait que le sujet n'est pas sa propre origine. Cette condition (ou incondition) de créature ne conduit justement à aucune origine, raison pour laquelle Lévinas parle d'une « créature, mais orpheline de naissance ou athée ignorant sans doute son Créateur [...] » (AE, pp. 165-6). S'il arrive à Lévinas de décrire la psyché, c'est-à-dire l'âme, en termes d'inspiration, c'est que pour Lévinas il y a souffle ou vie, dans la mesure où il y a trace, c'est-àdire cet avoir toujours déjà été affecté par Autrui (le fait que « je suis noué aux autres avant d'être noué à mon corps »). On peut dire alors, pour cette raison, que Lévinas va plus loin que Nietzsche. Ici la vie n'est pas l'indéconstructible de la généalogie : elle a elle-même une généalogie. ${ }^{26}$

Si la vie procède de la rencontre, elle n'est alors plus une seule force (et une force aveugle) qui se désire elle-même, comme l'affirme Nietzsche dans la « Seconde considération inactuelle ». Là, on ne peut plus tout à fait dire que Lévinas radicalise le projet nietzschéen puisqu'il déjoue la définition de la vie comme force. En fait, comme Nietzsche, mais pour de toutes autres raisons et

ouverte à l'être par quoi le monde est offert à l'interprétation. Même si l'ouverture à l'être n'est en rien une connaissance de l'être, en elle, pour Lévinas, se joue toujours un rapport de prise (et donc de savoir, même si non théorique) sur le monde. Ainsi, Alphonso Lingis écrit-il : " Heidegger thinks of our existence being open as being in an openness - it is in the sense of the hand that has leeway to move. Lévinas in this work presents the first movement of animation as an opening of the subject upon a space filled with air before being filled, or emptied by the light. The openness upon the air is not an intention or an apprehension but inspiration, our substance being open to the core » ("Otherwise than being or beyond essence », Kluwer Academic Publishers, 1991. Traduit en anglais par Alphonso Lingis, p. XXVIII).

26 Cette mise en question de la vie comme principe va dans le sens de la thèse qu'élabore Barbara Stiegler dans « Nietzsche et la critique de la chair ». Pour Barbara Stiegler, Nietzsche ne propose pas une affirmation inconditionnelle de la vie et du corps mais une critique de la chair (à savoir de ce qui fait qu'un corps est vivant, affectable, qu'il est une « chair affectable capable de s'éprouver elle-même en vie », Stiegler, 2005, p. 31). Cette thèse est tout à fait intéressante car elle montre que le défaut des lectures vitalistes de Nietzsche (et du vitalisme en général) est de s'en remettre à une conception désincarnée de la vie. Par ailleurs, la thèse de Stiegler rejoint l'articulation que nous proposons entre souffrance éthique et genèse de la vie : non seulement, pour Stiegler, afin de penser la chair, il faut s'attacher à la dimension passive de la souffrance, mais il faut aussi penser la sensibilité en rapport à une extériorité radicale : "Toute compréhension autoaffective de l'affect, qui enferme le sentir dans la sphère 'intime' de l'immanence, oublie que la passion et l'affect sont d'abord ce qui nous expose à l'extériorité comme telle » (ibid., p. 150). C'est là exactement la description que fait Lévinas de la relation entre sensibilité et rencontre éthique. En d'autres termes, pour Stiegler, non seulement Nietzsche n'est pas un vitaliste, mais sa conception de la vie est dérivée de cette compréhension de la chair comme exposition à l'extériorité. 
dans un tout autre sens, Lévinas pense bien la vie en termes d'infinité, mais cet infini ne procédant pas d'un manque, la vie ne se déploie pas seulement comme force. Pour Lévinas en effet, la vie qui procède de la rencontre est une « vie sans mort, vie de l'Infini » (AE, p. 223). Cet infini ne procède toutefois d'aucune idée de l'éternité, mais bien, encore, de la condition de créature ou de l'avoir été toujours déjà affecté. Dans la mesure où Autrui se présente en excès sur tout phénomène, le « pour Autrui » engage le moi par delà ses limitations. Par Autrui, la vie n'est pas à la mort (pas à la limite que constitue la mort), mais par delà la mort. La vie inspirée par Autrui n'est pas une vie définie négativement en relation à la finitude, à son manque constitutif, mais une vie qui vit de ce qui l'excède; une vie dont le cœur bat de l'autre. C'est pourquoi, pour Lévinas, la vie n'est pas seulement une force qui se désire ellemême. Elle ne vit pas seulement de la vie et de son manque constitutif. Pour Lévinas, il y a vie - respiration - à même une inspiration qui porte ailleurs. «La respiration est transcendance » (AE, p. 278) écrit Lévinas, elle vient de l'Autre, de l'ouverture au temps qu'il rend possible.

On peut alors observer comment, tout en radicalisant le projet nietzschéen, Lévinas retourne l'idée de force qui le soutient. En effet, tandis que la vie procède de l'autre par qui la vie est « un vie sans mort, une vie de l'Infini », la subjectivité n'est pas seulement par Autrui, elle est, de par l'excès d'Autrui, un « pour l'Autre ». Or, c'est bien ce pour qui déjoue l'idée que la vie ne serait qu'une force en expansion pour reprendre les termes qu'utilisait le jeune Lévinas dans ses «Quelques réflexions sur la philosophie de 1'hitlérisme ». Procédant de l'autre, elle est aussi et déjà dans le " pour l'autre »; inspiration, elle est aussi et déjà expiration. Paradoxalement, son infini (cette « vie sans mort ») est ce qui l'expose à la finitude; il est ce par quoi elle s'éprouve dans sa vulnérabilité. Naissant du « pour» qui inspire mais par là même blesse, la vie devient finie, vulnérable ou sensible, dans le rapport à Autrui, c'est-à-dire par le fait même qu'elle porte l'infini. La vie comme inspiration naît de ce porter l'autre qui est aussi un mourir pour lui. Ainsi, la " vie sans mort » est-elle paradoxalement ce par quoi la vie est aussi exposée au caractère irréductible de la finitude : « L'homme, demande Lévinas, n'est-il pas le vivant capable du souffle le plus long dans l'inspiration sans point d'arrêt et dans l'expiration sans retour? » (ibid., pp. 278-9).

\section{Conclusion : d'une compassion à l'autre}

Nous avons vu jusqu'ici que la question de la compassion chez Lévinas (entendue comme « souffrance pour Autrui ») n'allait pas dans le sens de 
la critique nietzschéenne de la compassion. La souffrance pour Autrui ne correspond chez Lévinas ni à une forme d'apitoiement ni à un déni de la vie. Au contraire, la souffrance éthique permet de penser la genèse de la vie sans s'en remettre à un principe ultime. Elle permet de penser la condition de créature sans s'en remettre à un créateur. Mais cette idée de vie créée nous permet d'aller plus loin dans le rapport entre Nietzsche et Lévinas. Comme cela a été vu par plusieurs commentateurs, Nietzsche n'est pas seulement critique de la compassion. S'il est vrai que dans « Les voies du créateur $»,{ }^{27}$ on lit une exhortation à la solitude (« Rentre dans ta solitude, ô mon frère, avec ton amour et ton vouloir créateur », Z, p. 104), la critique que Nietzsche fait à l'amour du prochain ne se fait pourtant pas au profit de la solitude mais de « l'amour du lointain » (Z, « De l'amour du lointain », p. 102). ${ }^{28}$ Or, le lointain, n'est-il pas justement celui que Lévinas appelle le « prochain » ? Pour Lévinas en effet, Autrui est irréductible, il n'a pas de commune mesure avec moi. Dans ce sens, Autrui est le prochain dans la mesure même où il est le lointain, c'està-dire le non assimilable. Mais la question ici n'est pas d'assimiler Nietzsche à Lévinas. Il s'agit plutôt de comprendre que la souffrance éthique va dans le sens d'une autre compassion à laquelle Nietzsche n'est pas étranger lorsqu'il en appelle à l'amour du lointain. En effet, si l'on suit la piste ouverte par Barbara Stiegler dans " Nietzsche et la critique de la chair », chez Nietzsche « la passion et l'affect sont d'abord ce qui nous expose à l'extériorité comme telle ». ${ }^{29}$ Il n'y aurait pas « d'amour du lointain » si la vie ne vivait que de son manque constitutif, si la vie n'était qu'une force aveugle en expansion. C'est parce que la vie vit d'hétéro-affection qu'elle ne s'enlise pas dans sa seule force anonyme et aveugle, qu'elle excède sa dimension tragique. Dans ce sens, l'incarnation (l'hétéro-affection qui caractérise la chair) est l' « incondition » de l'amitié pour reprendre ici un mot propre à Lévinas, de cet amour du lointain où l'autre est affirmé dans son étrangeté. Dans cette situation, non seulement la compassion ne réduit pas l'autre au Même, non seulement elle ne revient ni à l'économie nihiliste de la pitié ni à son calcul rédempteur (centré uniquement sur le sujet), mais elle permet de penser une forme de mit-leiden, de « souffrir avec » qui porte au-delà de soi et de l'autre, au-delà de la complaisance dans laquelle pouvait enliser la souffrance tragique

27 NIETZSCHE, F. « Ainsi parlait Zarathoustra » (dorénavant abrégé Z). Paris : Gallimard, 1985. Traduit en français par Maurice de Condillac.

28 Pour une lecture exhaustive de la question de la compassion chez Nietzsche, et en particulier de la possibilité de penser une éthique de Nietzsche (au-delà de la réduction de Nietzsche au rôle de généalogiste), voir l'article de Frazer (Frazer, 2006).

29 Déjà cité note 25. 
ou la fascination esthétique pour la souffrance. Ainsi, parce que la souffrance éthique provient d'une hétéro-affection qui ouvre au-delà, il est possible de la penser au-delà d'un cadre normatif, comme ce qui porte l'homme au-delà de lui-même, de son confinement. Sur un ton qui n'est somme toute pas si différent de l'élan lévinassien lorsqu'il affirme dans « Autrement qu'être » que " L'humanisme ne doit être dénoncé que parce qu'il n'est pas suffisamment humain » (AE, p. 203) Nietzsche écrit « De 1'amour du prochain »: " Que l'avenir, que les choses les plus lointaines soient pour toi les causes de ton labeur d'aujourd'hui ; en ton ami tu aimeras le Surhumain qui est la raison de ton être » $(\mathrm{Z}, 102)$.

\section{Bibliographie}

BERGO, B. "The Flesh Made Word; Or The Two Origins ». In : BERGO, B., STAUFFER, J. (éds.). Nietzsche and Lévinas. After the death of a certain God, New York : Columbia UP, 2008.

BLANCHOT, M. «L'écriture du désastre ». Paris : Gallimard, 1980.

CALIN, R. « Lévinas et l'exception du soi ». Paris : Puf, 2005.

EDGAR, A. "The art of useless suffering ». Medecin, Health Care and Philosophy, 10, 2007.

FRANK, D. «L'un pour l'autre. Lévinas et la signification ». Paris : Puf, 2008. . « Nietzsche et l'ombre de Dieu ». Paris : Puf, 1998.

FRAZER, M. « The compassion of Zarathustra : Nietzsche on sympathy and strenght ». Review of Politics, 68, Nr. 1, 2006.

LEVINAS, E. «La souffrance inutile». In : Entre nous. Essais sur le penser-à-l'autre. Paris : Grasset, 1991.

Payot, 1997.

. (1934). «Quelques réflexions sur la philosophie de l'hitlérisme ». Paris:

. (1947). « De l'existence à l'existant». Paris : Vrin, 1993.

. (1961). « Totalité et Infini ». LP, SD.

(1974). «Autrement qu'être ou au-delà de l'essence ». LP, SD.

LINGHIS, A. (1981). Preface. In : «Otherwise than being or beyond essence ». Kluwer Academic Publishers, 1991. Traduit en anglais para Alphonso Lingis.

MARION, J. L. « De surcroît ». Paris : Puf, 2001.

NIETZSCHE, F. (1872). « Naissance de la tragédie ». Paris : Gallimard, 1986. Traduit en français par Michel Haar, Philippe Lacoue-Labarthe et Jean-Luc Nancy. Édition de Giorgio Colli et Mazzino Montinari.

. (1873). "Considérations inactuelles », La gaya scienzia, Janvier 2012. Traduit en français par Henri Albert, édition électronique.

. «Ainsi parlait Zarathoustra ». Paris : Gallimard, 1985. Traduit en français par Maurice de Condillac. 
. (1987). «La généalogie de la morale ». Paris: GF, 2002.

. (1895). «L'antéchrist ». Trad. Robert Rovini. Paris : Benoît Jacob, 1967.

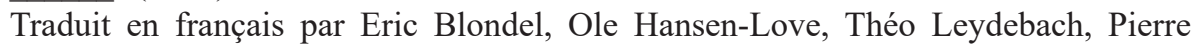
Pénisson.

OMBROSI, O. «L'umano ritrovato ». Milano : Marietti, 2010.

RICOEUR, P. «La souffrance n'est pas la douleur ». In : Autrement, Souffrances, Nr. 142, 1994 repris dans Souffrance et douleur. Autour de Paul Ricoeur. Paris : Puf, 2013 (Dir. Claire Marin et Nathalie Zaccai-Reyners).

STIEGLER, B. « Nietzsche et la critique de la chair. Dionysos, Ariane, le Christ ». Paris : Puf, 2005.

WHITE, R. «Lévinas, the philosophy of suffering and the ethics of compassion ». The Heythrop Journal, pp. 111-123, 2012.

ZAOUI, P. « La traversée des catastrophes. Philosophie pour le meilleur et pour le pire ».

Paris : Seuil, 2010. 